\title{
PATERNIDAD E IDENTIDAD MASCULINA EN COSTA RICA: EL SOBREDIMENSIONAMIENTO DEL MANDATO DE LA PROVEEDURÍA ${ }^{1}$
}

\author{
Mauricio Menjívar Ochoa \\ M.Sc. Ciencias Políticas \\ Estudiante Doctorado en Historia \\ Universidad de Costa Rica
}

\begin{abstract}
RESUMEN
El presente trabajo presenta los resultados parciales de una investigación de campo sobre el tema de la paternidad en la Costa Rica actual. El artículo centra su interés en la explicación del mandato masculino de la proveeduría y en las implicaciones que éste tiene sobre la forma en que los hombres conciben su paternidad. También indaga en los efectos de este mandato sobre el poco involucramiento de los hombres en las tareas de tipo reproductivo y, aún más, sobre la manera en que contribuye a precarizar el ejercicio mismo de la paternidad.
\end{abstract}

Descriptores: Paternidad. Masculinidad. Relaciones de género. Costa Rica. Siglo XX.

\section{INTRODUCCIÓN}

La discusión y posterior aprobación de la "Ley de paternidad responsable” en Costa Rica en el año 2001, ${ }^{1}$ ha contribuido a develar la problemática expresada en el hecho de que muchos hombres son genitores sin cumplir una función paterna alguna, entendiendo la genitura como mera aportación biológica a la gestación. Al volverse la paternidad centro del debate, también se ha puesto en evidencia una cierta diversidad de criterios y estereotipos en relación con la 
función paterna, muchos de los cuales develan un gran desconocimiento sobre la temática y la incipiente investigación sobre una base empírica.

En términos generales, existe una tendencia a definir la paternidad a partir de un desfase entre el padre socialmente requerido y lo que los hombres de nuestra época están dispuestos a cumplir. En esta dirección, algunos han señalado que "los hombres” están fallando, pues “...no han sabido comprender cuál es su lugar en el mundo actual en relación con este tema..." ( $L a$ Nación, 17/ 5/ 2000: 8A). Otras posiciones tienden a equiparar el "machismo" al fenómeno de la "paternidad irresponsable" (La Prensa Libre, 31/8/2001, pág.13), lo cual pareciera asociar la "responsabilidad" con un rasgo que se desprende de nuevas formas de ejercicio de la paternidad o formas "no machistas".

¿Guardan este tipo de ideas una relación con la forma en que los hombres entienden su paternidad? ¿Están los hombres malinterpretando las exigencias societales del sistema con relación a la paternidad? ¿En qué clave social debe entenderse la problemática de la paternidad responsable? O, más aún, considerando las aspiraciones en relación con el deber ser de la paternidad: ¿Es la paternidad concebida a partir del término responsable, la solución acabada al tema de la genitura disociada de la función paterna?

A partir de estas y otras cuestiones el presente trabajo busca exponer algunos de los resultados, que son parte de una investigación más amplia, ${ }^{2}$ propiciando un acercamiento a cuáles son las concepciones masculinas sobre la paternidad, particularmente las referidas a la proveeduría y a las labores de tipo reproductivo. En este sentido, y basado en la evidencia empírica recopilada en nuestra investigación, el primer apartado discute sobre el concepto de "responsabilidad" con el propósito de explicar su significación dentro de los mandatos masculinos tradicionales sobre la paternidad. Con ello se pretende alertar a las tomadoras y 
tomadores de decisiones sobre el uso del término "paternidad responsable", con el cual, a nuestro parecer, no puede avanzarse en la resignificación de la paternidad patriarcal. El segundo apartado busca profundizar la comprensión sobre la forma en que el mandato de la proveeduría moldea la identidad masculina y las actitudes de los hombres hacia su participación en las labores reproductivas del cuido de niños y niñas.

\section{LA MASCULINIDAD Y EL MANDATO DE LA PROVEEDURÍA}

El antropólogo David Gilmore (1994) en su sistemático intento por explicar la forma en que es construida la masculinidad en diversas culturas, encontró que en la concepción que hombres y mujeres de diferentes pueblos tienen al respecto de la "verdadera virilidad", adquieren especial relevancia "tres requerimientos morales". Estos requerimientos, si bien podían variar en intensidad, cobran el carácter de imperativos. Según Gilmore, “...para ser un hombre en la mayoría de las sociedades... uno debe preñar a la mujer, proteger a los que dependen de él y mantener a los familiares..." (Gilmore 1994: 217).

Si bien la existencia de esta tríada, que lleva a definir al sujeto que la ejerce como "el varón preñador-protector-proveedor", tiene implicaciones más profundas en relación con la perpetuación del orden social (Gilmore 1994: 217 y subs.), para nuestros efectos esta gran constatación empírica ameritaría varias observaciones más restringidas. En primer término, que esta definición de virilidad se sustenta en la división sexual del trabajo que asigna a mujeres y a hombres funciones sociales excluyentes, con las consabidas desigualdades de género. En segundo lugar, que dada esta división social del trabajo, esta tríada debe entenderse como una construcción que las sociedades erigen como una inducción de los hombres en 
función de la sobrevivencia del grupo. ${ }^{3}$ Es decir, la virilidad, bajo este punto de vista y como tendencia general, no se ocupa de preñar como un fin en sí mismo (es decir, como mera genitura), sino que se encuentra también vinculada a la producción económica y, con ella, a la proveeduría. ${ }^{4}$ Lo anterior lleva a plantear la preeminencia que dentro del proyecto asignado socialmente a la masculinidad tienen, tanto la paternidad como la proveeduría. En otros términos, estos son rasgos imbricados y constitutivos en el hecho de ser hombre en sociedades patriarcales. La línea explicativa del estudio de Gilmore no tiene como propósito indagar el fenómeno de la genitura sin involucramiento paterno entre un sector de la población de hombres, lo cual plantea que esta es, para los efectos de este estudio, una explicación parcial para la comprensión de la paternidad. ${ }^{5}$

Sin embargo, y teniendo presente tales reservas, los datos de nuestra investigación aportan algunos elementos para considerar que entre una parte de la población de hombres existe una tendencia concordante con lo señalado por Gilmore. En este sentido, para algunos de los entrevistados existen dos hitos estrechamente vinculados y que resultan definitorios para "sentirse un hombre". En primer lugar se encuentra el hecho de ser padre, así como la conformación de su propia familia. Efectivamente, una importante proporción de los hombres entrevistados sintió que ya era hombre cuando nació el primer hijo $(28,6 \%)$, cuando supo que iba a ser padre o en un sentido similar, cuando tuvo la responsabilidad de ser padre. Para otro 4,3\%, formar un hogar fue un hecho de gran relevancia en esta dirección.

El segundo hito de gran importancia para otra parte de los hombres es el tema del trabajo: el 18,8\% se sintió hombre cuando comenzó a trabajar o a aportar económicamente al hogar, y otro 5,8\% cuando asumió completamente la responsabilidad económica del hogar. ${ }^{6}$ 
Una segunda indagación al respecto de lo que hace a una persona ser hombre, esta vez hecha por medio de preguntas cerradas, ${ }^{7}$ ofrece un resultado similar: aquello que en primera instancia hace a una tercera parte de los hombres entrevistados ser definidos como hombres es formar una familia, ${ }^{8}$ mientras que ser padre lo es para otro $17 \%$. Para otro $17 \%$, si bien estos elementos no representaban aspectos de primer orden de prioridad, si eran elementos valorados. Estos dos factores ocupaban un segundo orden de importancia para otro $17 \%$ en ambos casos, en el hecho de ser hombre.

Por otra parte, para más de una tercera parte de los entrevistados, un elemento de primer orden de importancia que lo hace a uno ser hombre es el primer trabajo o el éxito laboral, mientras que para otro $25 \%$ este era un segundo elemento de prioridad, sumado a otro $9 \%$ que consideraba que ganar el primer sueldo era definitorio en este sentido. En otras palabras, desde la concepción cultural de los entrevistados el hecho de hacerse hombre está marcado por ser padre o tener un hijo, ${ }^{9}$ la formación de una familia y el trabajo, son elementos fundamentales y definitorios de la masculinidad.

Este tipo de consideraciones pueden llevar a preguntarse si algunos estudios realizados sobre el tema de la masculinidad, no habrán dado un peso sobredimensionado a la actividad ocupacional, como “...fuente principal de la identidad del hombre moderno..." (Gomariz 1997: 33). En este sentido, en una encuesta nacional sobre familias realizada en Chile, se preguntó a hombres y mujeres “¿Cuál es el área más importante de su vida?”, ante lo cual se detectó una respuesta masculina “....notablemente diferente a la femenina: la proporción más alta de varones se refirió a su trabajo o estudio, mientras la más alta de mujeres se refirió a la vida familiar..." (citado por Gomariz 1997). De lo anterior se colige la existencia de dos tipos 
de aspiraciones distintas, entre lo que destaca que el área familiar no es de tanto interés para los hombres.

Sin embargo, una interpretación distinta a la expuesta en el párrafo precedente, consideraría que el trabajo o actividad ocupacional entre una parte de los hombres, debe dimensionarse a la luz del proyecto familiar y de relación de pareja, en la medida que culturalmente el trabajo remunerado está en función de la sobrevivencia del grupo familiar. Si bien este pareciera una especie de matiz, en realidad tiene implicaciones importantes pues no plantearía el tema de la identidad ocupacional de los hombres como factor que está exclusivamente en función de su propio proyecto individual-ocupacional, como si estuviera en función de sí mismo. Es fundamental entender, siguiendo nuevamente a Gilmore (1994), que “...los códigos y normas morales de la cultura animan a la gente (a veces con premios y castigo psicológicos, en vez de materiales) a perseguir unos fines sociales, al mismo tiempo que satisfacen sus propios deseos personales..." (Gilmore 1994: 220). Es decir, que la cultura patriarcal plantea mecanismos que reconcilian las metas individuales con las del grupo, de forma tal que a la vez que los hombres obtienen reconocimiento público y capacidad de dominio a partir de su actividad ocupacional, también se encuentran en función de la sobrevivencia familiar mediante lo que se ha designado como proveeduría. Esta se plantea como una forma de estar en función de los otros, si bien claramente distinta a la socialmente asignadas a las mujeres.

Podría parecer contradictoria esta afirmación a la luz de la gran cantidad de hombres que evaden la pensión alimentaria, sobre lo cual hablaremos más adelante. Sin embargo, existe alguna evidencia empírica para sostener que el mandato de la proveeduría se cumple especialmente con aquellas personas cubiertas por la relación de pareja actual de los hombres 
(Ver Menjívar 2002: Cap. 4). Es decir, al menos una buena parte de los hombres tiende a involucrarse económicamente con niños y niñas acogidas en el marco de la relación de pareja en las que ellos se encuentran inmersos. Esto significa que tienden a quedar más desprotegidos los niños y niñas de relaciones de pareja anteriores, los cuales ya no parecieran encontrarse cubiertos por el término de familia en este sentido restrictivo. Esta incongruencia dentro del mandato masculino de la proveeduría pareciera ser un factor fundamental a tomar en cuenta al momento de promover paternidades involucradas.

Teniendo clara esta paradoja, y considerando a la familia en sentido restringido, uno de los mecanismos sociales que pareciera operar entre algunos hombres como código de conducta que vincula la genitura y la proveeduría, a la vez que reconcilia las metas individuales con las grupales, es el concepto de responsabilidad. Para poner este concepto en perspectiva histórica habría que señalar que las sociedades occidentales, especialmente desde el siglo XIX, han impuesto que los hombres se vean “...forzados a trabajar durante todo el día fuera de la casa..." (Badinter 1993: 148), reduciendo notablemente el contacto entre los padres de familia urbanos y sus hijos. Esto convierte a los padres en personajes comparativamente más lejanos que en períodos previos, cuyos quehaceres, la mayor parte de las veces, son un misterio para sus hijos. En este modelo se encuentra por un lado, “...la mujer, madre y ama de casa; por otro, el hombre... encargado de la alimentación..." (Badinter 1993: 148), ubicándolo como responsable de la proveeduría ante su familia.

En este sentido, no es casual que la característica más difundida dentro de la valoración que los entrevistados tienen con relación a su personalidad como hombre sea la responsabilidad: es la primera característica más valorada para un $23,2 \%$ y la segunda característica más valorada para otro $10,3 \%{ }^{11}$ 
Cabe mencionar, que dentro de aquellos entrevistados que no se encontraban casados (solteros, separados en unión libre) era más alto el porcentaje de los que se adherían a la responsabilidad como código actitudinal masculino (aproximadamente el 50\%), que entre los que se encontraban casados $(30 \%)$. De hecho, la mayoría de los que llegaron a inscribir a sus hijos o hijas se encontraban en Unión Libre. Esto aporta evidencia que demuestra que no todos los hombres tienen la idea de que “...los niños nacidos fuera del matrimonio, y aún en unión libre, son responsabilidad de las mujeres...", ${ }^{12}$ como algunos han afirmado (La Nación, 17/ 5/ 2000: 8A).

Por otra parte, la mayoría de los hombres entrevistados comenzó a trabajar a muy temprana edad: un 41,8\% entre los 10 y los 14 años y un 43,3\% entre los 15 y los 19 años. Esta situación de trabajo a edad temprana es especialmente acentuada entre aquellos que valoran de su personalidad de hombres el hecho de ser trabajadores y ser responsables. Según el testimonio de algunos de los entrevistados, las condiciones adversas en las que han comenzado a laborar también tienen incidencia en la construcción de estos valores. Una parte de los hombres entrevistados, que en su mayoría tienen ocupaciones de baja cualificación, con baja escolaridad y bajos salarios, han salido a trabajar a temprana edad impulsados por la familia, ante el requerimiento de la sobrevivencia, lo cual pareciera incidir en el reforzamiento de su papel de responsabilidad en cuanto a la proveeduría. ${ }^{13}$

Ante lo anotado en relación con las concepciones sobre la responsabilidad, no extrañará que de aquellos entrevistados que dijeron haber escuchado sobre la Ley de Paternidad Responsable $(59,2 \%)$, prácticamente la totalidad estuviese de acuerdo con ella. La mayoría planteó su acuerdo señalando simplemente que le parecía "muy bien (53,3\%); sin embargo, otros plantearon su acuerdo porque era una ley que favorecía ya sea a los niños y niñas $(6,7 \%)$, 
a la las mujeres $(8,9 \%)$ tanto a niñas y niños como a las madres $(2,2 \%)$, o incluso a los hombres para demostrar su paternidad $(2,2 \%)$. Otros señalaron que esta era una ley "para que el hombre se haga o lo hagan más responsable" (15,6\%).

Lo anterior se ve confirmado por dos encuestas realizadas por el Instituto de Investigaciones sobre Población (IDESPO) de la Universidad Nacional de Heredia, ${ }^{14}$ durante la fase de discusión del proyecto de ley de Paternidad Responsable en la Asamblea Legislativa. Según estos resultados, una gran proporción de hombres estaba "muy de acuerdo" o "de acuerdo" con el proyecto de ley: el 88,5\% en el Gran Área Metropolitana, y un 96\% en el Área Metropolitana de San José, frente al 78,7\% y el 87\%, respectivamente, en el caso de las mujeres (IDESPO 2000: 59). Esto nuevamente da pie a pensar que dentro de una importante proporción de hombres la responsabilidad es un código actitudinal anclado socialmente.

Ahora bien, cuando una parte de la población de hombres huyó del mandato de la responsabilidad, es decir de la proveeduría económica, se designó a este tipo de hombres como padres irresponsables. Es fundamental avanzar en el estudio de este tipo de masculinidades, al respecto de lo cual discutiremos algunos aspectos en el siguiente apartado. Sin embargo, aquí resulta interesante señalar el caso de uno de los entrevistados, que se presentó al Registro Civil a solicitar una prueba de ADN por supuesta duda de paternidad. ${ }^{15}$ Sin embargo, durante la entrevista correspondiente a esta investigación este hombre dio a entender, no sin cierto cinismo, que en realidad el niño del cual estaba solicitando prueba de ADN, realmente era hijo suyo. Su motivo para solicitar la prueba consistía en una venganza con la madre del niño por haberse acogido a la Ley de Paternidad Responsable, por una parte, y por no haber invertido en su destino final el dinero que él le daba para que ella planificara (y de ahí el nacimiento del niño). Aún más, este hombre señaló que "seguro ahorita le caía otra notificación”, pues además 
de este hijo de cinco meses, tenía otro de dos meses. Solamente se encontraba a la expectativa de que la madre de este otro hijo decidiera acogerse a la ley. En ninguno de los casos había hecho aporte económico alguno, no conocía al niño de dos meses, mientras que a aquel por el cual se encontraba en el Registro, apenas lo había visto tres veces en sus cinco meses de vida.

Resulta sumamente importante señalar que este hombre valora altamente ser "perro" o "noviero" y pelear, como características definitorias de su personalidad de hombre. Para este hombre con fuertes características de la masculinidad hegemónica, las concepciones de masculinidad no pasan por los códigos actitudinales relacionados con la responsabilidad o la seriedad, ni con el interés en la familia. En casos como este es evidente la existencia de una disociación entre el ejercicio de la sexualidad y sus implicaciones sobre la reproducción, así como entre la reproducción y el ejercicio de la función paterna. Parte del resultado de esta disociación es la transferencia de las consecuencias de las propias prácticas reproductivas a la mujer, la cual "no se cuidó", muestra de un claro ejercicio irresponsable, no sólo de su sexualidad, sino de las consecuencias sobre la genitura.

Estos códigos de conducta masculina contrastan notablemente con algunos hombres que se consideran a sí mismos del tipo "responsable". Uno de estos, al ser inquirido en relación con las características que más valoraba de su personalidad como hombre sentenció: “...La responsabilidad es la base: quien no es responsable no es hombre..."

Debe señalarse, que el discurso de la responsabilidad como principio de autoidentificación identitaria de los hombres todavía tiene, desde nuestra perspectiva, algunos vacíos de conocimiento en relación con sus verdaderos efectos prácticos y cotidianos. En este sentido, cabe señalar que uno de los elementos que este estudio buscaba indagar estaba referido a si aquellos hombres que solicitaban pruebas de ADN al Registro Civil funcionaban 
con las características del hombre genitor que rehuye la responsabilidad sobre su conducta reproductiva. Sin embargo, esto no fue posible determinar con toda seguridad. Llama la atención que tres de los entrevistados se autodefinieron como "responsables" y, al mismo tiempo, señalaron como motivo de solicitud de la prueba de $\mathrm{ADN}$, "...no estar seguros de que el hijo fuera suyo..." Este parece ser un pretexto, no poco común, entre quienes rehuyen su responsabilidad paterna, sin embargo, no fue posible saber los resultados de las pruebas de ADN y determinar si eran o no los padres. En todo caso, tal posibilidad nos plantea la necesidad de estar atentos ante la diversidad que existe en las construcciones masculinas; lo cual es una confirmación de la necesidad de hablar de masculinidades (en plural), así como de profundizar la investigación que parta de esta diversidad para descifrar adecuadamente los códigos culturales que les subyacen.

Quisiéramos concluir este apartado, señalando que uno de los elementos que sobresale entre la evidencia mostrada, alude directamente a que la responsabilidad es parte de las concepciones identitarias de un segmento considerable de los hombres entrevistados. También resulta claro que estas concepciones se encuentran afincadas en la construcción tradicional (patriarcal) de la masculinidad. Por ello, la responsabilidad pareciera ser uno de los mecanismos sociales que vincula la genitura y la función económica del padre, por lo que la proveeduría se encuentra a la base de la imagen paterna de una parte importante de los entrevistados.

Todo pareciera apuntar, a que una parte de los hombres han entendido claramente cuál es el mandato que la época le impone y, a partir de este, han asimilado su lugar en el mundo actual, de ahí que Joseph-Vicent Marqués (1997) plantee que “...el sentido de la responsabilidad es oficialmente masculino..." (Marqués 1997: 22), o al menos de un tipo 
preponderante de masculinidad. De igual manera existen otros hombres que, sin esgrimir el discurso de la responsabilidad cumplen con su papel de proveedor.

Sin embargo, es conveniente tomar en cuenta que entre otros hombres este mandato tiende a poseer límites muy claros, los cuales son definidos por la concepción de "familia" que estos manejan (Ver Menjívar 2002: Cap. 4). Desde esta concepción la familia pareciera estar restringida a la relación de pareja en la que se encuentran, diluyendo su importancia cuando se trata de niños, niñas y mujeres de relaciones anteriores. Esto significa que en este último caso, tales hombres tienden a desinvolucrarse considerablemente como padres, dejando descubiertas las necesidades de sus hijos e hijas y, por lo tanto, sobrecargando a las mujeres quienes asumen la crianza de niños y niñas. Lo anterior podría llevar a pensar que el sentido de la responsabilidad masculino desde este mandato, además de contradictorio, brinda a muchos hombres una gran libertad para establecer nuevas relaciones, sin tener que sobrellevar las consecuencias de la genitura.

Otra parte de la población de hombres, difícil de cuantificar, responde a un discurso masculino que tiende a disociar sexualidad y genitura, así como genitura y función paterna, engrosando las filas de la denominada "paternidad irresponsable". Esta heterogeneidad existente en cuanto a códigos masculinos, es un punto medular que debe ser ponderado al momento de definir acciones tendientes al fomento de paternidades cumplidoras en lo económico. Podría pensarse que el discurso de la paternidad responsable es parte del acervo cultural de una parte de la población de hombres, lo cual amerita un abordaje diferenciado en relación con el que se daría a hombres con concepciones culturales afincadas en la doble disociación antes planteada. La indagación al respecto de este tipo de masculinidad resulta fundamental para avanzar en su conocimiento. 
Sin embargo, existe evidencia que permite sostener que el mandato masculino de la responsabilidad, bajo los márgenes que tiene la definición de familia, adquiere culturalmente un matiz economicista, en la medida en que tiende a centrarse en la proveeduría, ante lo cual cabe preguntarse ¿Qué implicaciones tiene tal sesgo sobre la percepción que tienen los hombres acerca de la paternidad?. Por otra parte, de existir un sobredimensionamiento del papel de proveedor en la función paterna, caben al menos otras interrogantes. En primer término: ¿Qué consecuencias tiene tal sobredimensionamiento en relación con otras funciones paternas como la del cuido?, y en segundo lugar ¿Qué sucede cuando los hombres no pueden cumplir adecuadamente con la proveeduría? En el siguiente apartado nos abocamos a discutir estas y otras cuestiones relativas a la proveeduría.

\section{PROVEEDURÍA VERSUS CRIANZA EN LA FUNCIÓN PATERNA}

Una de las premisas básicas de la división de trabajo que han propuesto las feministas es la que sostiene, que “...la división entre el hombre proveedor económico y la mujer ama de casa está basada en una percepción complementaria de roles para hombres y mujeres, que son $<<$ diferentes pero iguales $>>$..." (Moser 1995: 52). Caroline Moser, siguiendo a Mackintosh, señala que la “...penetración del capitalismo occidental con su separación histórica de la producción y la reproducción es la que ha producido esa división artificial y su refuerzo ideológico..." (Mose 1995: 52).

Esta división y su peso sobre la cultura, contribuyen a explicar la importancia que ha tenido el mandato de la proveeduría en la conformación de la identidad masculina de una buena parte de los hombres. Este fenómeno tiene dos implicaciones que interesa destacar aquí. 
En primer lugar debe mencionarse que al ser la identidad masculina elemento fundante de la identidad paterna, se produce un fuerte ligamen entre la paternidad y la proveeduría a grado tal que esta última sirve en una parte de la población entrevistada como parámetro evaluador de la paternidad y la masculinidad. En esta dirección, el punto número 2.1 de este apartado busca plantear algunos elementos que muestran este ligamen. Sin embargo, teniendo claridad al respecto de la existencia de patrones que fomentan en los hombres la genitura sin involucramiento paterno, se exponen algunos elementos de orden cultural que fragilizan tal involucramiento.

En segundo lugar, según lo anotado por Moser, el mandato de la proveeduría tiende a desligar a los hombres de las tareas del cuido. Precisamente ubicándonos en el terreno de lo reproductivo, la segunda parte de este apartado (2.2) indagará en las actitudes de los entrevistados hacia algunas de las tareas del cuido en que los padres tienden a involucrarse en mayor o menor medida. Esta revisión será vista a partir de una perspectiva crítica a la concepción patriarcal de la complementariedad, que justifica la división sexual del trabajo en la que los hombres se ubican en el plano de la proveeduría y las mujeres en el ámbito reproductivo, concepción cultural que se mantiene a pesar de que estas últimas han operado cambios sustantivos mediante su incorporación al mercado de trabajo.

\subsection{El mandato de la proveeduría en la función paterna}

Como ha quedado de manifiesto en el apartado número 1 de este artículo, la proveeduría es una función fundamental dentro del involucramiento paterno de muchos hombres, en tanto deviene en elemento constitutivo de su masculinidad. La Encuesta Nacional sobre 
Masculinidad realizada por el Centro Nacional para el Desarrollo de la Mujer y la Familia en el año de 1997 constató, entre otros elementos, que el 31,2\% de los hombres costarricenses consideraba que llevar el dinero al hogar era una situación que le hacía sentir plenamente hombre. En el caso de las mujeres, esta situación hacía sentir plenamente mujer a apenas un 8.9\% de la población, tendiendo la mayoría dela población femenina a reforzar el mandato masculino de la proveeduría (CMF 1997: 46).

En este sentido, como se puede apreciar en el Cuadro 1, casi un $60 \%$ de los entrevistados en el marco de nuestra investigación, consideraron que la función más importante de un padre dentro de la familia es llevar el dinero. Sin embargo, el dinero no sólo define una parte medular de la función paterna en el marco del patriarcado sino que define o, más aún, mide la calidad de la paternidad misma. En esta dirección el capitalismo patriarcal establece una relación directa entre cantidad de dinero y calidad de la paternidad. Efectivamente, desde una definición "positiva", a mayor cantidad de dinero aportada por los padres al hogar, mejor paternidad. De ahí que aproximadamente la tercera parte de los hombres entrevistados señalaran que cuanto más dinero lleve al hogar un padre, mejor padre se es.

\section{AQUÍ VA CUADRO 1}

Sin duda alguna, la posibilidad de generación de ingresos tiene un peso desmedido en la autoestima paternal, pues desde una definición negativa, más de la quinta parte de los entrevistados considera que un hombre que no tiene trabajo no puede ser un buen padre.

También es posible observar que otros entrevistados están en desacuerdo con la rigidez de este rol, lo que muy posiblemente se encuentra asociado a los cambios que han venido 
gestando las mujeres a partir de su inserción laboral. Si bien es cierto que tal inserción ha significado una doble y hasta una triple jornada de trabajo con profundas implicaciones sobre las mujeres (Moser 1995: 50-63), también ha generado una resignificación de la división del trabajo. Parte del resultado de esta resignificación es que el aporte económico de una gran cantidad de mujeres al hogar no sea subestimado o devaluado pues, según constató la Encuesta Nacional de Masculinidad (1997: 30-31), para el 66,4\% de los hombres los ingresos que aportan las mujeres son "muy importantes" y para otro 23,4\% son "importantes", sucediendo algo muy similar con relación a la valoración que las mismas mujeres hacen de su aporte. ${ }^{17}$

Ahora bien, la claridad cultural del mandato de la proveeduría "...no significa que la función proveedora de la paternidad esté exenta de contradicciones y confusiones..." (Rodríguez y Lázaro 2001: 18), como bien señalan Rodríguez y Lázaro en un estudio realizado para la CEPAL. Una de estas contradicciones radica, siguiendo la argumentación de estos autores, en el incremento de las demandas por pensión alimentaria en Costa Rica. En 1990, el número de demandas por pensión alimentaria en vigencia ascendía a 23,805, adicionándose otras 8,396 ese mismo año. Ya para 1998, “...las demandas vigentes sumaban 41,890 y se presentaron 15,383 nuevas. En otras palabras, sólo en este último año había en curso 57,273 pensiones alimentarias...” (Rodríguez y Lázaro 2001: 19). Bajo el supuesto que de que existiera un solo caso de pensión alimentaria por familia, Rodríguez y Lázaro estiman que en el año de 1998 “...habría por lo menos $6.59 \%$ de hogares costarricenses en los que se registra una pensión alimentaria demandada o en ejecución...” (Rodríguez y Lázaro 2001: 19).

Mientras que para muchos hombres la proveeduría es un mandato incuestionable, para otra parte no pareciera ser un asunto automático con el que se cumple sin más, existiendo la 
necesidad de mecanismos coercitivos que procuren garantizarla. En vista de esta situación, nos interesa señalar algunos elementos de tipo cultural que fragilizan el involucramiento paterno.

Uno de estos elementos que fragilizan el involucramiento paterno se produce cuando el desmedido peso de la proveeduría como función paterna no da cabida a otro tipo de funciones en las que los padres se podrían involucrar. Efectivamente, la afirmación que prescribe que a mayor cantidad de dinero se lleve al hogar mejor padre se es, o la que afirma que el hombre que no tiene trabajo no es un buen padre, como señalamos con anterioridad, encierran una equiparación entre proveeduría y paternidad, que convierte a ambos términos en sinónimos. Siguiendo esta lógica patriarcal, para los entrevistados no tiene sentido hacerse cargo de sus hijos cuando los hombres no pueden cumplir con el mandato de la proveeduría, de ahí que algunos (8.4\% de los entrevistados) consideren que estar sin empleo es una buena razón para que los hombres no vean por sus hijos (Ver Cuadro 2).

En este mismo sentido, Muñoz y Calderón (1998) encontraron en su estudio sobre embarazo adolescente en comunidades pobres, que “...asumir la paternidad es problematizado por las dificultades que encuentran los adolescentes para ejercer su papel de proveedores y protectores...", pues el temor de no poder asumir adecuadamente este encargo, “...provoca que los hombres prefieran evadir su responsabilidad antes de enfrentarse al fracaso..." (Muñoz y Calderón 1998: 129), lo cual se constituye en una suerte de privilegio masculino.

\section{AQUÍ VA CUADRO 2}

Un segundo elemento que fragiliza el involucramiento paterno en la crianza de los hijos e hijas alude a la ya mencionada desvinculación que hacen algunos hombres entre su 
comportamiento sexual y las consecuencias de este comportamiento sobre la genitura, por una parte, y entre la genitura y el involucramiento paterno, por otra. En este sentido, como se observa en el Cuadro 2, poco más de un $10 \%$ de los entrevistados consideran que el hombre no debe hacerse cargo de los hijos e hijas que nazcan de una aventura sexual.

Un tercer elemento que fragiliza el involucramiento deriva de la incapacidad que tienen algunos (alrededor de un 10\% de los entrevistados) de deslindar la propia paternidad respecto de la relación que tienen con la madre de su hijo o hija. Efectivamente, estos hombres consideran que si el hombre no ama a la madre de su hijo o hija, es comprensible que no se haga cargo de ellos/as. Esta concepción se produce al visualizar a la madre y al hijo como una sola unidad, respecto de la cual el hombre se relaciona de manera marginal o simplemente no se relaciona, lo que contribuye a precarizar o a anular la relación paternal. Esta misma incapacidad de deslindar las relaciones se refleja en que para aproximadamente una cuarta parte de los entrevistados el hombre no debe de dar dinero para sus hijos si la madre ya tiene otra relación.

Resulta importante señalar que en principio manejamos la hipótesis que tendía a apuntar a que estas concepciones se hallarían especialmente difundidas entre aquellos hombres que llegaron al Registro Civil a solicitar pruebas de ADN por duda o por rechazo de paternidad, ${ }^{18}$ si se les comparaba con los que llegaron a inscribir a sus hijos e hijas, ya sea por que fueron citados, ${ }^{19}$ o porque llegaron a inscribir a sus hijos por su propia cuenta. Sin embargo se ha podido constatar que es precisamente entre los que llegaron a inscribir donde se encuentran especialmente ancladas (Ver Cuadro 3). Así, el que un hombre inscriba a su hijo o hija no es sinónimo de que vaya a ejercer una paternidad plenamente involucradas si se presentasen circunstancias como las que se han venido analizando. 
La presencia de estos tres tipos de concepciones culturales pareciera apuntar a la existencia de condiciones paternales poco maduras con un gran potencial destructivo respecto del involucramiento paterno en una parte de la población entrevistada. Este es un nuevo indicador de la necesidad de reforzar en la socialización masculina, la posibilidad de establecer vínculos paternos más allá de la relación que los hombres tengan con la madre de sus hijos e hijas.

\section{AQUí VA EL CUADRO 3}

\subsection{La Función paterna de la crianza: involucramiento en las tareas del cuido}

Como se señaló al principio de este apartado el que la identidad de una buena parte de los hombres entrevistados esté signada por la proveeduría, tiene como correlato la concepción de que las mujeres deben procurar las condiciones reproductivas y las tareas de cuido. De ahí que, prácticamente la mitad de los entrevistados consideren que en la familia el hombre debe llevar el dinero y la mujer criar a los hijos.

Y ni aún la tendencia a la creciente incorporación de las mujeres al trabajo remunerado ha logrado, hasta hoy, hacer que para los hombres se rompa esta división de esferas. Es decir, muchas mujeres tienen que enfrentar la doble jornada, mientras que una buena proporción de los hombres sigue sin involucrarse mayor cosa en las labores reproductivas. En este sentido, según el propio testimonio de las tres cuartas partes de los hombres y del $80 \%$ de las mujeres entrevistadas por la Encuesta Nacional de Paternidad (CMF 1997: 34-38), los hombres participan muy poco o simplemente no participan en las tareas domésticas, incrementándose 
levemente su participación únicamente en el caso de que su compañera o esposa trabaja remuneradamente. Según dicha encuesta, entre el $89,6 \%$ y el $92 \%$ de los hombres nunca o muy pocas veces participa en el lavado de la ropa $;^{20}$ entre el $72,8 \%$ y el $82,7 \%$ nunca o muy pocas veces cocina, y entre el $84 \%$ y el 91,7\% nunca o muy pocas veces plancha (Ver Cuadro 4), siendo todas estas actividades de gran importancia en la creación de condiciones adecuadas no solo para la propia sobrevivencia sino para la de hijos e hijas. Aún más, entre 58 y 68 hombres de cada 100 nunca o muy pocas veces cuida a los niños y niñas de sus hogares. Sólo en las tareas de la compra del diario y la reparación de la casa los hombres se incorporan en mayor medida (Ver Cuadro 4). ${ }^{21}$

\section{AQUÍ VA EL CUADRO 4}

Joseph-Vincent Marqués (1997), en su abordaje sobre la teoría patriarcal de la complementariedad, contribuye a explicar esta fuerte tendencia a la segmentación de labores. Según Marqués, la teoría de la complementariedad, influida por formas “...menos abiertamente misóginas del cristianismo oficial o institucionalizado..." (Marqués 1997: 28), al menos como discurso deja de poner énfasis en la culpabilización de la mujer por la entrada del mal al mundo y deja de insistir en la inferioridad de la mujer. Ahora, “...hombre y mujer serían de igual dignidad pero diferentes. Esa diferencia supondría precisamente una mutua necesidad resuelta por la complementariedad de las cualidades de uno y otro sexo..." (Marqués 1997: 28). Pero la lectura que se hace de esta supuesta complementariedad es “...que la mujer es el complemento del hombre y no que éste deba ser, o deba ser también, el complemento de la mujer..." (Marqués 1997: 29). La teoría de la complementariedad lleva, 
entre otras cosas, a que el hombre espere que una mujer realice aquellas tareas de las que él no puede ocuparse en tanto que a él le "corresponde" la proveeduría.

Como puede apreciarse en el Cuadro 5, existen una serie de tareas de tipo reproductivo que corresponden a la función del cuido, que una parte de los hombres considera que no les corresponde desempeñar, pues son de responsabilidad materna. En este sentido, según el 30\% de los entrevistados, los hombres no deben cambiar pañales, mientras que alrededor de una quinta parte piensa que no es responsabilidad de los hombres alistar a los niños y niñas para ir a la escuela. Otro tanto considera que es asunto de las madres asistir a las reuniones de "padres" de familia, llevar a los hijos y a las hijas al médico o levantarse en la noche cuando las y los bebés lloran. Algunas de las tareas del cuido en que una mayor proporción de los entrevistados piensa que los hombres deben involucrarse, tienen que ver con dar medicinas (según el $81,7 \%$ de los entrevistados), ayudar a hijos e hijas en las tareas escolares $(84,5 \%$ ) y acompañarles a las actividades deportivas $(87,3 \%)$.

Es preciso anotar que la posibilidad de que los hombres realicen en mayor medida unas tareas (dar medicinas, ayudar en tareas escolares o acompañar a las actividades deportivas) sobre otras (levantarse en la noche cuando los bebés lloran o, peor aún, cambiar pañales) no resulta de la mera casualidad. Pierre Bourdieu (2000), en su estudio sobre La dominación masculina, ha señalado que existe una disposición que inclina a los hombres “...a dejar a las mujeres las tareas inferiores y las gestiones molestas y mezquinas..." (Bordieu 2000: 48). En este sentido, el desvelo y, aún más el manejo de la materia fecal de los bebés, tienen una clara diferencia en relación con otras actividades como la recreación y la formación de valores que, son en las que los hombres más se visualizan involucrados (Ver Menjívar 2002: Cap. 3). 


\section{AQUÍ VA EL CUADRO 5}

Es necesario señalar, por otra parte, que durante el proceso de entrevista, a muchos hombres les era difícil tomar una posición en lo relativo a si era sólo asunto de las mujeres llevar a hijos e hijas al médico y llevarles a la escuela. Efectivamente, al solicitárseles tomar una de dos opciones opuestas (estar de acuerdo o en desacuerdo) con relación a estos temas, estos hombres señalaron que ellos lo harían si el horario de su trabajo se los permitiera; sin embargo estas actividades (cuido y trabajo) por lo general chocaban. A este respecto, si bien algunos hombres tienden a justificar su falta de involucramiento en el cuido con la necesidad de cumplir su rol de proveedor, tampoco puede negarse que en ciertos casos el horario laboral es un referente objetivo que imposibilita a algunos hombres a participar en ciertas tareas de orden reproductivo. En este sentido, no es de extrañar que muchos hombres para garantizar la sobrevivencia de su familia deben laborar fuera de su casa extensas jornadas. A este respecto debe señalarse, sin embargo, que las mujeres siguen trabajando mayor cantidad de horas (PNUD 1995).

La anterior situación genera una importante contradicción cuando se considera la creciente expectativa social de que los hombres se involucren en el ámbito doméstico. En este sentido, retomando a Rodríguez y Lázaro (2001), “...por una parte, se sigue considerando a la proveeduría, tanto por hombres como por mujeres, como el valor fundamental de la paternidad y del ser hombre, pero simultáneamente ésta (la proveeduría) se ha convertido en un antivalor asociado al abandono y a la despreocupación por los hijos y por el hogar..." (Rodríguez y Lázaro 2001: 19). Resulta claro que resulta necesario resolver estas contradicciones que surgen 
cuando, como señalan Rodríguez y Lázaro, “...la función proveedora sirve como medida para evaluar la función paterna..." (Rodríguez y Lázaro 2001: 19).

En relación con el cuido es importante destacar, por último, la importante proporción de hombres, que al menos en el plano de las actitudes, considera que las tareas de cuido de los hijos no son asunto exclusivo de las mujeres. Como puede observarse en el Cuadro 5, dependiendo del tipo de tareas existe entre un $69 \%$ y un $87,3 \%$ de los hombres que visualizan a su género involucrándose en el cuido. Tal pareciera, que a la par del combate de la proveeduría como única medida para evaluar la función paterna, debe de explorarse entre los hombres la posibilidad de este otro tipo de involucramiento paterno.

\section{CONSIDERACIONES FINALES}

Como hemos anotado es importante tener en cuenta que las concepciones que configuran el mandato mediante el cual una gran proporción de los hombres se erige en proveedor, es un mandato que paralelamente produce una exclusión masculina de las tareas del cuido. Este mandato tiene un fuerte anclaje en la identidad masculina (es decir, sobre la manera en que se perciben como hombres), impregnando fuertemente la forma en que muchos de los hombres viven su paternidad. En otros términos, el mandato patriarcal de una paternidad centrada en la proveeduría lleva aparejado el mandato de exclusión de los hombres de las tareas del cuido. Adicionalmente debe tomarse en cuenta que el hecho de que muchos hombres no asuman la proveeduría no se acompaña necesariamente de un involucramiento en la esfera reproductiva, lo cual redunda en mayores cargas de trabajo para las mujeres. 
El efecto perverso del desmedido peso de la proveeduría en la identidad paterna de algunos hombres se manifiesta cuando tal mandato va en detrimento de otro tipo de vínculos. Estas condiciones que fragilizan el involucramiento paterno es lo que hemos designado preliminarmente como inmadurez de las condiciones paternas, para señalar la falta de construcción de otros vínculos que permitan un involucramiento paterno de carácter más integral.

La falta de madurez se refleja en el hecho de que la relación padre-hijo/hija se encuentra supeditada a los vaivenes de la relación de pareja (padre-madre), de la cual pareciera depender en alto grado. La necesidad de independizar estos dos tipos de relaciones se hace necesaria para que una eventual ruptura en la relación de pareja y un eventual reemparejamiento de la mujer, no conduzcan a una ruptura del vínculo que los padres tienen con sus hijos e hijas.

La falta de madurez de las condiciones paternas parecieran estar asociadas a una concepción existente entre algunos hombres, tendiente a visualizar a mujeres y niños y niñas como una especie de unidad en la que él se presenta no plenamente involucrado en todas las funciones paternas, sino más bien como un sujeto marginal. Esta inmadurez puede ser atribuible en buena medida al desmedido peso que tiene la proveeduría en la identidad paterna, la cual no va acompañado de un mayor desarrollo de otro tipo de vínculos.

Un reto más complejo para el involucramiento paterno se puede visualizar aquellos casos en los que niños y niñas son producto de relaciones catalogadas como de "aventura sexual" por sus genitores. Tanto entre aquellos hombres con condiciones paternas de poca madurez como entre los que disocian su sexualidad de sus consecuencias sobre lo reproductivo, las vías para el involucramiento paterno pasan por la reeducación y, en cualquier caso, por el reforzamiento de la normativa existente en materia de Paternidad Responsable y Pensiones Alimentarias. 


\section{BIBLIOGRAFÍA}

Badinter, Elisabeth. 1993. XY. la identidad masculina. Colombia: Editorial Norma.

Bordieu, Pierre. 2000. La dominación masculina. Barcelona: Anagrama.

Centro Nacional para el Desarrollo de la Mujer y la Familia. 1997. Masculinidad, salud reproductiva y paternidad responsable. Encuesta nacional. San José: Centro Nacional para el Desarrollo de la Mujer y la Familia / ByS consultores.

Gilmore, David. 1994. Hacerse hombre. Concepciones Culturales de la masculinidad. España: Paidós.

Gomariz, Enrique. 1997. Introducción a los estudios sobre Masculinidad. San José: Centro Nacional para el Desarrollo de la Mujer y la Familia.

Instituto de Estudios en Población. 2000. La población costarricense del Gran Área Metropolitana frente a los tiempos de cambio y los roles entre hombres y mujeres. Heredia: IDESPO, Universidad Nacional.

Marqués, Joseph-Vincent. 1997. "Varón y Patriarcado". En: Valdés, Teresa y Olavaria, José, eds., Masculinidad/es, Poder y Crisis. Santiago de Chile: Ediciones de las Mujeres, No.24, Isis/FLACSO-Chile.

Menjívar Ochoa, Mauricio. 2001. "La paternidad responsable: un análisis desde lo masculino". En: Vega, Isabel, comp. Inscripción de los hijos e hijas de las madres solteras: ¿Una cuestión de paternidad responsable?. San José: Instituto de Investigaciones Psicológicas, Universidad de Costa Rica.

. 2002. Actitudes masculinas hacia la paternidad: entre las contradicciones del mandato y el involucramiento. San José: INAMU, Colección Teórica No.2.

Moser, Caroline. 1995. Planificación de género y desarrollo. Teoría, práctica y capacitación. Lima-Perú: Red Entre Mujeres, Ediciones Flora Tristán.

Muñoz, Sergio y Calderón, Lucía. 1998. Maternidad y paternidad: las dos caras del embarazo adolescente. San José: Centro Nacional para el Desarrollo de la Mujer y la Familia.

PNUD. 1995. Informe sobre Desarrollo Humano 1995. México: PNUD/Harla, México.

Rodríguez, María Elena y Lázaro, Luis. 2001. "La paternidad responsable en Costa Rica: Una tarea pendiente". México: CEPAL, 9 de julio 2001.

\section{PERIÓDICOS}

La Nación, 17/ 5/ 2000, p. 8A.

La Prensa Libre, 31/ 8/ 2001, p. 13.

\section{NOTAS}

\footnotetext{
${ }^{1}$ Este artículo forma parte de una investigación más amplia, la cual fue publicada en el libro: Mauricio Menjívar, Actitudes Masculinas hacia la Paternidad. San José: INAMU, 2002.
} 
${ }^{1}$ Esta ley (No. 8101) fue aprobada por la Asamblea Legislativa de Costa Rica en marzo de 2001. Para un análisis de algunas de las implicaciones de esta ley ver: Menjívar 2001.

${ }^{2}$ Aquí se recoge la mayor parte de los capítulos primero y segundo de la investigación: Menjívar Ochoa, Mauricio. Actitudes masculinas hacia la paternidad: entre las contradicciones del mandato y el involucramiento. 1 ed. San José: INAMU, 2002 (Colección Teórica No.2). En esta investigación colaboraron Roger Esquivel y Mikel Otxotorena y fue realizada en el marco de la Unidad de Investigación del Instituto Nacional de las Mujeres de Costa Rica. Tal Unidad pertenece al Área Especializada de Información de dicho Instituto. Para este estudio fueron entrevistados 71 hombres en las Oficinas Regionales del Registro Civil, de las siete cabeceras de provincia de Costa Rica. Todas las entrevistas se llevaron a cabo en el mes de marzo de 2002. En términos generales, el perfil socioeconómico de estos hombres es de baja escolaridad y bajos ingresos económicos. Cabe mencionar que en esta investigación es central el concepto de involucramiento paterno, mismo que alude a la posibilidad de los hombres de implicarse o estar incluidos en las diversas funciones paternales y significa a la vez participación y compromiso. Se trata de un concepto que pretende conducir la indagación acerca de cuáles son los aspectos en que los hombres se ven como partícipes en relación con la crianza de los hijos y las hijas y, por exclusión, en cuáles no se ven a sí mismos implicados (Menjívar 2002: 15).

${ }^{3}$ Sin duda alguna, se trata de un aporte a la sobrevivencia desde un ángulo distinto al desarrollado desde la feminidad que, si bien tradicionalmente se encuentra centrado en lo reproductivo, cada vez más se desplaza a lo productivo.

${ }^{4}$ En este estudio el tema de la "protección", segundo componente de la tríada, no aparece con tanta fuerza.

${ }^{5}$ Parte de las reservas que tiene el estudio de Gilmore para contribuir con la comprensión de la paternidad es que si bien estudia sociedades occidentales "modernas", presta mayor interés en sociedades no occidentales consideradas por cierta rama de la antropología como "primitivas".

${ }^{6}$ Otros hitos en el paso a sentirse hombre son la primera relación sexual o de pareja $(8,7 \%)$, la autonomía personal (4.3\%) y la vida militar para algunos hombres nicaragüenses $(4,3 \%)$.

${ }^{7}$ Como mencionamos, las preguntas con opciones predeterminadas por quien investiga ("preguntas cerradas") tienen la utilidad de contribuir a contrastar hipótesis. Sin embargo tienen la limitación de encasillar a las personas entrevistadas y pueden, eventualmente, pasar por alto cuestiones sustantivas en la percepción de los entrevistados. Por esta razón la presente investigación combina "preguntas abiertas" -aquellas en donde no existen preguntas precodificadas- en cuestiones claves sobre lo que significa ser hombre y padre, con preguntas cerradas.

${ }^{8}$ Se le solicitó a los entrevistados que establecieran un orden descendente de prioridades entre aquellos elementos que ellos consideraban más importantes en el hecho de ser hombre. De ahí que se hable de elementos de un primer orden de importancia o que en primera instancia son importantes y así de manera sucesiva.

${ }_{9}^{9}$ En algunos casos los entrevistados señalaron que ser padre hace a una persona ser hombre, mientras que otros apuntaron que "tener un hijo". No es posible dentro de los márgenes de esta investigación determinar si existe o no algún matiz simbólico en esta distinción. Sin embargo podría resultar de interés indagar al respecto.

${ }^{11}$ Existen otras características altamente valoradas como ser trabajador $(14.5 \%)$, la seriedad $(7,2 \%)$ y ser entregado a la familia $(5,8 \%)$. Si bien cabe una mayor indagación sobre la forma en que los hombres están entendiendo el término "seriedad", la evidencia arrojada por nuestra investigación sugiere que es muy posible que, como código de conducta masculino, la seriedad implica no tomarse las cosas a la ligera en la vida, sino que asumirse de manera sensata y formal. Otros códigos de conducta que resultaría interesante estudiar son la sinceridad $(7,2 \%)$, la humildad $(7,2 \%)$, la honestidad $(15,4 \%$ como segunda opción altamente valorada), que parecieran valores culturales socialmente apreciados que complementen la autoimagen masculina sobre lo que poco se sabe en nuestro medio.

${ }^{12}$ El marco de la aplicación de la Ley es precisamente los hijos/as nacidos de una unión libre o de una relación no cubierta bajo el matrimonio.

${ }^{13}$ Debe aclarase que esto no significa necesariamente que hombres de otro perfil sociodemográfico no puedan adherirse a estos códigos masculinos.

${ }^{14}$ Se trata de una encuesta telefónica a 400 personas en el Gran Área Metropolitana y otra personal a 200 personas en el Área Metropolitana de San José (IDESPO 2000).

${ }^{15}$ La Ley de Paternidad Responsable establece que si el padre declarado por las Mujeres que se acogen a dicha Ley, se presenta al Registro Civil y no acepta o duda de la paternidad, se le dará una única cita de forma gratuita para realizar la prueba de ADN en el laboratorio de la Caja Costarricense de Seguro Social y determinar si es el padre o no (Menjívar 2002: 126). 
${ }^{17}$ Cabría preguntarse si muchos hombres visualizan el aporte de sus esposas o compañeras como un reparto de la carga de la proveeduría y, siendo así relativicen la visión rígida que define al padre a partir de la cantidad de su aporte económico. Sin embargo, existen indicios de que en lo afectivo se presenta una cierta diversificación de las posibilidades, pues la afectividad no se centra exclusivamente en la proveeduría, lo que contribuye a entender porqué otra gran cantidad de los padres entrevistados no consideran que si un padre no tiene empleo no es un buen padre o que no necesariamente la cantidad de dinero está asociada a una mejor paternidad. En este sentido, la preocupación que muchos hombres depositan en la formación de valores, en la espiritualidad y en ser un padre ejemplar, parecieran actuar como agentes que compensan el obsesivo y enorme peso cultural que tiene la proveeduría en el involucramiento paterno. Véase a este respecto: Menjívar 2002: Cap. 3.

${ }^{18}$ Debe tomarse en cuenta que en total se entrevistaron 8 hombres que solicitaron pruebas de ADN por duda de paternidad y únicamente a un hombre que solicitó prueba de ADN por rechazo de paternidad.

${ }^{19}$ El citatorio es un mecanismo ideado por el Registro civil, previo a la notificación que establece la Ley de Paternidad Responsable.

${ }^{20}$ La proporción más alta de los que nunca o muy pocas veces realizan tareas domésticas son hombres cuya compañera o esposa no trabajaba remuneradamente.

${ }^{21}$ En el caso concreto de las compañeras o esposas de los entrevistados de nuestra investigación, es importante señalar que el 64,8\% se dedicaba al trabajo en el hogar como actividad exclusiva, y sólo un 19,7\% tenía una ocupación remunerada, lo cual reafirma la división sexual del trabajo desde la óptica tradicional. La información relativa al $12,7 \%$ de las mujeres no fue suministrada por los entrevistados. Otro 2,8\% de las mujeres era estudiante. 